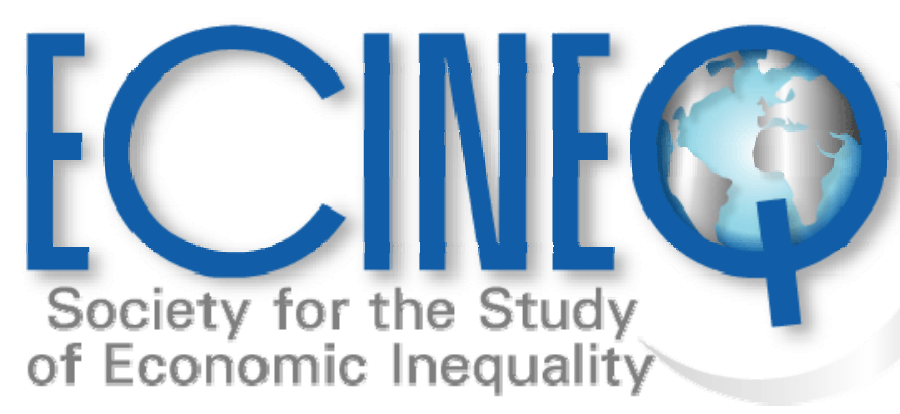

Working Paper Series

\title{
A model of economic geography with demand pull and congestion costs
}

Olga Alonso-Villar 


\title{
A model of economic geography with demand pull and congestion costs*
}

\author{
Olga Alonso-Villar ${ }^{\dagger}$
}

\begin{abstract}
This paper proposes a simple model of economic geography in which to derive analytical results when jointly considering two centrifugal forces - congestion costs together with the pull demand effect - within the Dixit-Stiglitz-Iceberg framework. In this vein, we develop a unified model with labor mobility that combines some of the features of Tabuchi (1998) with those of Forslid and Ottaviano (2003). We analytically show that when considering the effects of congestion costs, dispersion of economic activity is possible not only at high but also at low transport costs. This result corroborates previous numerical simulations conducted by Tabuchi (1998).
\end{abstract}

Keywords: R12, F12, F15, O18

JEL Classification: Congestion costs, demand pull, urban concentration, transport costs.

\footnotetext{
* Financial support from the Spanish Ministry of Education and Science via grant SEJ2005-07637-C02-01/ECON and from FEDER is gratefully acknowledged. I also want to thank Amelia Verdejo for helpful comments.

${ }^{\dagger}$ Correspondence address: Universidade de Vigo; Departamento de Economía Aplicada; Campus Lagoas-Marcosende s/n; 36310 Vigo; Spain. Tel.: +34 986 812507; fax: +34 986 812401; e-mail: ovillar@,uvigo.es
} 


\section{Introduction}

According to United Nations estimates, in 2003 48\% of the world's population lived in urban areas, a percentage which is expected to exceed $50 \%$ by 2007 . Hence, for the first time in history there would be more urban residents than rural. This interest in urban areas means that people and firms find advantages in carrying out their activities in a limited spatial area. In recent years, a great number of works have been focused on explaining the causes of this phenomenon. In this vein, within the New Economic Geography, the relevance of transport costs has been widely recognized in analyzing the location of economic activity. However, some shortcomings remain since the effects of transport costs reductions are not always the same in these models.

By using a framework where the industrial labor force is geographically mobile, while farmers are immobile and evenly distributed between locations, Krugman's (1991) seminal paper shows by numerical simulations that when manufacturing transport costs are low, firms tend to agglomerate in a single location. However, when manufacturing transport costs are high, firms are more interested in reaching the dispersed rural market, so that an even distribution of production between locations emerges. More recently, Forslid and Ottaviano (2003) develop an analytically solvable version, yielding the same results. ${ }^{1}$ Both papers emphasize the role of an immobile rural demand as the centrifugal force that encourages firms and workers to disperse between locations.

\footnotetext{
${ }^{1}$ Robert-Nicoud (2005) proves that the core-periphery model by Krugman and the model by Forslid and Octaviano are isomorphic.
} 
There is another line of studies, developed in the field of urban economics, which focuses instead on urban costs — mainly caused by pollution, commuting costs and housing consumption in large agglomerations - and yields different results. ${ }^{2}$ In this vein, by building a model where centrifugal forces stem from land consumption and commuting costs rather than from rural demand, Murata and Thisse (2005) analytically show that agglomeration is a stable equilibrium at high transport costs, while dispersion prevails at low transport costs. On the other hand, by conducting numerical simulations, Tabuchi (1998) shows that, when considering these intraurban costs together with the demand pull, dispersion emerges both at low and high transport costs. ${ }^{3}$ At low transport costs, consumers prefer to disperse to enjoy greater consumption of land (and lower commuting costs), while at high transport costs, the demand pull — which arises from immobile farmers who are geographically dispersed - is the force driving the result. ${ }^{4}$ It follows then that, when jointly considering both centrifugal forces, the effect of transport costs on the spatial distribution of economic activity is non-monotonic. ${ }^{5}$

The aforementioned bell-shaped relationship between the spatial distribution of production and transport costs has also been obtained in frameworks other than that of Dixit-Stiglitz-Iceberg. As a matter of fact, when using the approach of Ottaviano et al. (2002), which considers alternative assumptions about preferences and transportation costs, several papers yield the same result by considering an immobile demand

\footnotetext{
${ }^{2}$ Analyzing urbanized areas in the US between 1980-2000, Glaeser and Kohlhase (2004) point to an increase of over $280 \%$ in the average annual hours of delay, a percentage which rises to $450 \%$ in large cities. This fact together with an increase of $25 \%$ in real income per capita for the same period suggests an important loss due to congestion costs. Hardford (2006) considers additional damages caused by pollution.

${ }^{3}$ Forslid et al. (2002) find evidence of this relation between transport costs and overall concentration of manufacturing activities in Europe by using a CGE-model.

${ }^{4}$ Tabuchi (1998) only finds analytical results for two extreme cases: zero and infinite transport costs.

${ }^{5}$ This non-monotonic relationship is also found in other frameworks where the labor force is instead immobile. See, for example, Venables (1996) and Puga (1999).
} 
(farmers) together with a large variety of other centrifugal forces (see Picard and Zeng, 2005; and Tabuchi and Thisse, 2002).

The aim of this paper is to propose a simple model of economic geography in which to obtain analytical results when jointly considering two centrifugal forces - congestion costs together with the pull demand effect- within the Dixit-Stiglitz-Iceberg framework. In this vein, we develop a unified model with labor mobility that combines some of the features of Tabuchi (1998) with those of Forslid and Ottaviano (2003). Solvability is achieved by introducing congestion costs in such a way that they affect consumption rather than effective labor —unlike Krugman and Livas Elizondo (1996) and Murata and Thisse (2005) — thereby making the model more tractable. Apart from unifying different strands of work and finding analytical results, this paper shows the different roles played by immobile and mobile workers in explaining the location of economic activity.

The paper is organized as follows: Section 2 describes the assumptions of the model. The equilibrium is characterized in Section 3, and the main results are presented in Section 4. Finally, Section 5 draws the main conclusions.

\section{The model}

Consider a world consisting of two locations, labeled 1 and 2, and two sectors: agriculture and manufacturing. The first is perfectly competitive and produces a homogeneous good, while the second produces differentiated varieties under increasing returns to scale, and firms are assumed to compete in a monopolistic regime of the Dixit 
and Stiglitz (1977) type. In this economy there are $H$ high-skilled workers, who are freely mobile between locations, and $L$ low-skilled workers, who are immobile. Each individual supplies one unit of labor non-elastically. Agriculture only uses unskilled labor, while each firm in the manufacturing industry uses both skilled and unskilled labor.

\subsection{Preferences}

Consumers have Cobb-Douglas preferences over the agricultural good and a constantelasticity-of-substitution (CES) aggregate of manufacturing goods,

$$
U=z_{M}^{\mu} z_{A}^{1-\mu},
$$

where $\mu \in(0,1)$ denotes the manufacturing share, $z_{A}$ is consumption of the agricultural good and $z_{M}$ is consumption of the manufactures aggregate, which is defined by

$$
Z_{M}=\left(\sum_{i} Z_{i}^{\frac{\sigma-1}{\sigma}}\right)^{\frac{\sigma}{\sigma-1}},
$$

where $\sigma$ is the elasticity of substitution between any two varieties, $\sigma>1$. We assume that, in transporting manufacturing goods from another location, consumers incur transport costs that take the usual iceberg form. In particular, when a unit is delivered from the other location, the amount that arrives is only $1 / \tau, \tau \geq 1$. On the other hand, inside each city there are some additional costs, termed congestion costs, as a consequence of urban transport, pollution or housing prices, which means that the higher the city size, the higher the value of these costs. Congestion costs are also assumed to take the iceberg form. Thus, when a unit of a good is produced in, or arrives at, city $j$, any consumer living there can only obtain a proportion $1 /\left(H_{j}+L_{j}\right)^{\gamma}, \gamma \geq 0$, 
where $H_{j}$ and $L_{j}$ represent the number of high- and low-skilled workers, respectively, in location $j$. We therefore consider that congestion costs affect consumption rather than the effective labor force, which allows solvability of a model that jointly considers two different dispersion forces: one caused by an immobile demand and the other by congestion costs. ${ }^{6}$ Notice that, first, any individual living in the same city suffers the same congestion costs and, second, the higher the population size, the higher these urban costs.

Following Dixit and Stiglitz (1977), the price of the above aggregate for individuals living at location $j$ is:

$$
T_{j}=\left[n_{j}\left(p_{j j}\right)^{1-\sigma}+n_{k}\left(p_{k j}\right)^{1-\sigma}\right]^{1 /(1-\sigma)}, \quad j, k=1,2,
$$

where $p_{k j}$ is the price of any variety produced in location $k$ and sold in $j$ and $n_{k}$ is the number of varieties produced in location $k$.

\subsection{Agriculture}

Agriculture is perfectly competitive, producing a costlessly tradeable good that we choose as the numeraire. This sector is described by a constant returns to scale technology $F\left(L_{A}\right)=L_{A}$, where low-skilled labor is the only factor of production. We

\footnotetext{
${ }^{6}$ In Murata and Thisse (2005) and in Krugman and Livas Elizondo (1996), congestion costs are explicitly decomposed in housing and commuting costs. Since higher commuting costs in a location are compensated by lower housing prices (and vice versa), urban costs are equal for any individual living in the same city. Then, these costs can be summarized by the commuting costs at the edge of the city. Therefore, the consequence of considering these urban costs is that they affect the effective labor force in that city. However, none of these works jointly consider those urban costs with the pull of an immobile demand. On the other hand, Tabuchi (1998) considers a more general framework, with both commuting/housing and an immobile demand, but results are only obtained by numerical simulations.
} 
denote by $w_{A k}$ the wage paid in this sector in location $k$. All this means that $p_{A k}=w_{A k}=1$, where $p_{A k}$ denotes the price of this good. ${ }^{7}$

\subsection{The manufacturing industry}

As in Forslid and Ottaviano (2003) (F-O hereafter), we assume that the production of a single variety involves a fixed cost in terms of high-skilled labor (due for example to headquarter services or R\&D) and a marginal cost in terms of low-skilled labor, so that the cost function for any firm in $k$ is given by

$$
C_{k}=\alpha w_{k}+\beta x_{k}, \quad k=1,2,
$$

where $x_{k}$ is the number of units produced by this firm and $w_{k}$ is the high-skilled wage. ${ }^{8}$

Given the existence of fixed costs in terms of skilled labor, the number of firms in $k$ is $n_{k}=\frac{H_{k}}{\alpha}$.

\section{Solving the model}

Individuals in this economy must decide their consumption, taking into account that some manufacturing goods are produced in their city, but others have to be transported from outside. Total demand at location $j$ for any variety produced at location $k$ is given by:

\footnotetext{
${ }^{7}$ Low-skilled workers can receive the same salary in the two locations if the agricultural good is produced in both. For this to be the case, the aggregate demand of this good should be higher than the supply that a single location can offer. This implies that $\mu<\frac{\sigma}{(2 \sigma-1)}$.

${ }^{8}$ Skill heterogeneity between workers and differences in interregional mobility are the key assumptions in the core-periphery model proposed by F-O to achieve solvability.
} 


$$
Z_{k j}=\mu Y_{j} \frac{\left(p_{k j}\right)^{-\sigma}}{T_{j}^{1-\sigma}}, j, k=1,2
$$

where

$$
Y_{j}=w_{j} H_{j}+L_{j}
$$

represents the income at location $j$ and

$$
p_{k j}=p_{k} \tau\left(H_{j}+L_{j}\right)^{\gamma}, j \neq k
$$

is the price, including both transportation and congestion costs, paid by any individual located in $j$ for any good produced by a firm in $k$ charging price $p_{k}$. If the good is produced instead in location $j$ :

$$
p_{j j}=p_{j}\left(H_{j}+L_{j}\right)^{\gamma}
$$

The profit of a single firm in location $k$ can be written in terms of two demands, the demand of consumers located in $k$ and the demand of consumers from outside:

$$
\Pi_{k}=p_{k}\left(x_{k k}+x_{k j}\right)-\beta\left(x_{k k}+x_{k j}\right)-\alpha w_{k}
$$

where $x_{k j}$ represents the supply to location $j$, including the fraction of the good that melts away because of intercity transportation and urban costs, while $x_{k k}$ is the supply to its own location. The profit-maximization problem of this firm yields the following f.o.b. price:

$$
p_{k}=\beta \frac{\sigma}{\sigma-1}, \quad j=1,2
$$

since $w_{A k}=1$. As in F-O, this price does not depend on the firm's location, which makes the model more tractable. Monopolistic competition means that firms enter the market until profits become zero. This implies that the amount of any variety produced by a firm located at $k$ is given by: 


$$
x_{k k}+x_{k j}=\frac{\alpha(\sigma-1)}{\beta} w_{k}, \quad j, k=1,2
$$

Taking into account that $x_{k k}=Z_{k k}\left(H_{k}+L_{k}\right)^{\gamma}$ and $x_{k j}=Z_{k j} \tau\left(H_{j}+L_{j}\right)^{\gamma}$ and using expression (1) it follows that:

$$
x_{k k}+x_{k j}=\frac{\mu Y_{k} p_{k}^{-\sigma}\left(H_{k}+L_{k}\right)^{\gamma(1-\sigma)}}{T_{k}^{1-\sigma}}+\frac{\mu Y_{j} p_{k}^{-\sigma} \tau^{1-\sigma}\left(H_{j}+L_{j}\right)^{\gamma(1-\sigma)}}{T_{J}^{1-\sigma}} .
$$

On the other hand, by using expression (3), the price index can be written as:

$$
T_{k}=\left(H_{k}+L_{k}\right)^{\gamma}\left(\beta \frac{\sigma}{\sigma-1}\right)\left(n_{k}+n_{j} \phi\right)^{\frac{1}{1-\sigma}}
$$

where $\phi=\tau^{1-\sigma}$, so that production can be finally given by:

$$
x_{k k}+x_{k j}=\frac{\mu(\sigma-1)}{\beta \sigma}\left(\frac{Y_{k}}{n_{k}+n_{j} \phi}+\frac{Y_{j} \phi}{n_{k} \phi+n_{j}}\right) \text {. }
$$

Therefore, local demand faced by any firm increases with individuals' income level and decreases with the number of firms. It should be mentioned that both the market size effect and the effect of competition between firms are reduced by distance through parameter $\phi$.

From expressions (4) and (6), it follows that the wage rate at $k$ can be written as:

$$
w_{k}=\frac{\mu}{\alpha \sigma}\left(\frac{Y_{k}}{n_{k}+n_{j} \phi}+\frac{Y_{j} \phi}{n_{k} \phi+n_{j}}\right)=\frac{\mu}{\sigma}\left(\frac{Y_{k}}{H_{k}+H_{j} \phi}+\frac{Y_{j} \phi}{H_{k} \phi+H_{j}}\right) .
$$

For a given allocation of high- and low-skilled labor between locations, equations (2), (3), (5) and (7) characterize the f.o.b. price $\left(p_{k}\right)$, the quantity produced $\left(x_{k k}+x_{k j}\right)$, and the high-skilled wage $\left(w_{k}\right)$. By substituting (2) into (7) we obtain a system of linear equations in $w_{1}$ and $w_{2}$ - identical to that obtained by F-O - that can be analytically solved: 


$$
w_{k}=\frac{\mu / \sigma}{1-\mu / \sigma} \frac{\phi H_{k} L+\left[L_{K}\left(1-\mu / \sigma+\phi^{2} \mu / \sigma\right)+L_{j} \phi^{2}\right] H_{j}}{\phi\left(H_{k}^{2}+H_{j}^{2}\right)+\left[1-\mu / \sigma+\phi^{2}(1+\mu / \sigma)\right] H_{k} H_{j}} .
$$

Therefore, the equilibrium wages can be explicitly determined as a function of the distribution of labor between locations. It follows then that the way urban costs have been included in the analysis allows us to keep the same advantages of the aforementioned model in a framework which jointly considers two different centrifugal forces: the demand pull and congestion costs. In particular, it can be shown that if lowskilled labor is equally divided between both locations, the city with more high-skilled workers offers the highest (high-skilled) wage whenever that $\phi>\frac{1-\mu / \sigma}{1+\mu / \sigma}$. This is the result of two opposite forces. On one hand, an increase in the number of high-skilled workers raises the city income and therefore demand, which allows firms to pay higher salaries (equations (2) and (7)). On the other hand, it also allows the creation of new of firms (equation (7)), which induces a competition effect. The former prevails over the latter so long as transport costs are low enough.

\section{The results}

Once the number of firms, the prices, the quantities of goods and the wages have been determined for any given distribution of the population between both locations, we can analyze the location decision of individuals. As mentioned above, high-skilled workers are assumed to have higher geographical mobility. In particular, the low-skilled are immobile, while the high-skilled are freely mobile. As usual in this kind of model, we assume that individuals are short-sighted and they move to the location that offers the 
highest current utility. Skilled workers, however, do not move immediately, but there is a gradual process in response to increasing and decreasing changes in utility. In this vein, defining $h \equiv \frac{H_{1}}{H}$ as the proportion of skilled labor in location 1 , the time derivative of $h$ can be expressed by:

$$
\dot{h}= \begin{cases}W(h, \phi) & \text { if } 0<h<1 \\ \min \{0, W(h, \phi)\} & \text { if } h=1 \\ \max \{0, W(h, \phi)\} & \text { if } h=0\end{cases}
$$

where $W(h, \phi)$ is the indirect utility differential, which is proportional to the real wage differential $\frac{w_{1}}{T_{1}^{\mu}}-\frac{w_{2}}{T_{2}^{\mu}}$. Therefore, if location 2 offers a higher real wage, high-skilled workers will move from location 1 to location 2, and so forth until there is either full agglomeration of the skilled in location 2, or the two locations offer the same real wage.

\section{Sustainability of full agglomeration}

This section studies when full agglomeration in one location is a (locally stable) equilibrium of the economy. ${ }^{9}$ Let us assume that low-skilled workers are evenly distributed between both locations $\left(L_{j}=\frac{L}{2}\right)$ and that high-skilled labor is concentrated in location $2(h=0)$. It follows then that:

$$
\begin{gathered}
\frac{w_{1}}{w_{2}}=\frac{1-\mu / \sigma+(1+\mu / \sigma) \phi^{2}}{2 \phi} \text { and } \\
\frac{T_{2}}{T_{1}}=\left(\frac{H+L / 2}{L / 2}\right)^{\gamma} \phi^{\frac{1}{\sigma-1}} .
\end{gathered}
$$

\footnotetext{
${ }^{9}$ If full concentration is an equilibrium, it is also stable.
} 
Therefore, the real wage in location 2 is higher than that of location 1 if and only if:

$$
F(\phi) \equiv \frac{2 \phi^{1-\frac{\mu}{\sigma-1}}}{1-\mu / \sigma+(1+\mu / \sigma) \phi^{2}}>\left(\frac{H+L / 2}{L / 2}\right)^{\gamma \mu} .
$$

The most interesting case is that where $\sigma>1+\mu$ (the no-black-hole condition), which is assumed from now on. ${ }^{10} F$ looks as in Figure 1 , where $F(0)=0, F(1)=1$ and it has a maximum between 0 and 1 :

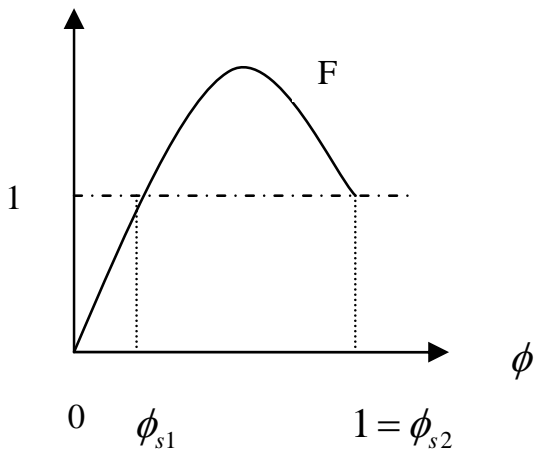

Case a)

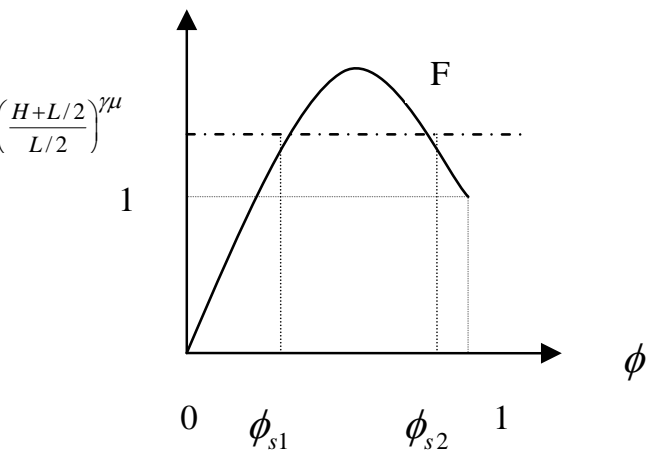

Case b)

Figure 1. Analysis of the "sustain points"

\footnotetext{
${ }^{10}$ If $\sigma-1 \leq \mu$, that is, if the agricultural good had a low weight in utility and manufactured goods were highly differentiated, $F$ would be a decreasing function which would tend to $\infty$ when $\phi$ were close to 0 , and would be equal to 1 at $\phi=1$. Therefore, if there were no congestion costs $(\gamma=0)$, concentration would be always equilibrium, as in F-O.
} 
If there were no congestion costs $(\gamma=0)$, full agglomeration would be a stable equilibrium for any value of $\phi>\phi_{s 1}$, i.e. where function $F$ is higher than 1 (case a). This means that full agglomeration would emerge as an equilibrium for low transport costs (i.e. for high values of $\phi$ ), as in F-O and Krugman (1991). However, when including urban costs in the model (case b), concentration is only possible for intermediates values of $\phi, \phi_{s 1}<\phi<\phi_{s 2}$. These critical points are given by the two cuts between curve $F$ and a horizontal line at height $\left(\frac{H+L / 2}{L / 2}\right)^{\gamma \mu}$ :

$$
\left(\frac{H+L / 2}{L / 2}\right)^{\gamma \mu}(1+\mu / \sigma) \phi_{s}^{2}-2 \phi_{s}^{1-\frac{\mu}{\sigma-1}}+\left(\frac{H+L / 2}{L / 2}\right)^{\gamma \mu}(1-\mu / \sigma)=0
$$

Note that congestion costs make the horizontal line in Figure 1 move up, so that instead of having one "sustain point", there are two. In other words, concentration cannot emerge as a possible equilibrium either at high or low transport costs. We can also prove that the higher the congestion costs, the lower the range of transport costs within which full agglomeration is possible. To sum up, we have analytically shown that full agglomeration is not a possible equilibrium for high transport costs, whether there are congestion costs or not. Furthermore, when considering these urban costs in the model, concentration at low transport costs becomes more difficult, so that this spatial configuration can only be reached at intermediate transport costs.

When analyzing the effects of the immobile and mobile labor forces in the model, we see that if $L$ is large as compared to $H,\left(\frac{H+L / 2}{L / 2}\right)^{\gamma \mu}$ approaches 1 , so that the range of transport costs that make concentration possible is close to that of F-O. In particular, the larger this demand, the easier it is to achieve concentration at low transport costs (high 
values for $\phi$ ), since the demand pull becomes the most effective centrifugal force, so that results mirror those of Krugman (1991). On the contrary, the larger the mobile demand, the more difficult it is to reach full agglomeration in one location, since congestion costs become a more important centrifugal force. In particular, these urban costs make it impossible to find agglomeration at low transport costs (high values for $\phi$ ). Numerical explorations of the above critical values suggest that the range of transport costs in which full agglomeration is a stable equilibrium shrinks with $\sigma$ and expands with $\mu$.

Symmetry breaking

Assuming that the immobile labor is equally distributed between the two locations, an even distribution of the high-skilled workers is always a spatial equilibrium, since real wages in both locations are equal. In this section we analyze when this equilibrium is (locally) stable.

It can be shown that the slope of $\mathrm{W}(\mathrm{h}, \phi)$ is negative at $h=0.5$ and, therefore, the symmetric equilibrium is stable if and only if:

$$
G(\phi) \equiv A \phi^{2}+B \phi+C>0,
$$

where

$$
\begin{aligned}
& A=(\mu+\sigma)[(H+L)(\sigma-1+\mu)+\mu(\sigma-1) \gamma H], \\
& B=-2\left[(H+L)\left((\sigma-1) \sigma+\mu^{2}\right)-\mu(\sigma-1) \sigma \gamma H\right], \\
& C=(H+L)(\sigma-\mu)(\sigma-1-\mu)+\mu(\sigma-\mu)(\sigma-1) \gamma H .
\end{aligned}
$$


Function $G$ is a convex parabola, $G(0)>0$ (if $\sigma>1+\mu$ ) and $G(1)>0 .{ }^{11}$ It can be shown that the minimum of this function is reached between 0 and 1 , if $0 \leq \gamma \leq 1$. Let us assume then that $0 \leq \gamma \leq 1$, since otherwise symmetry would always be a stable equilibrium, independently of the values of the remaining parameters.
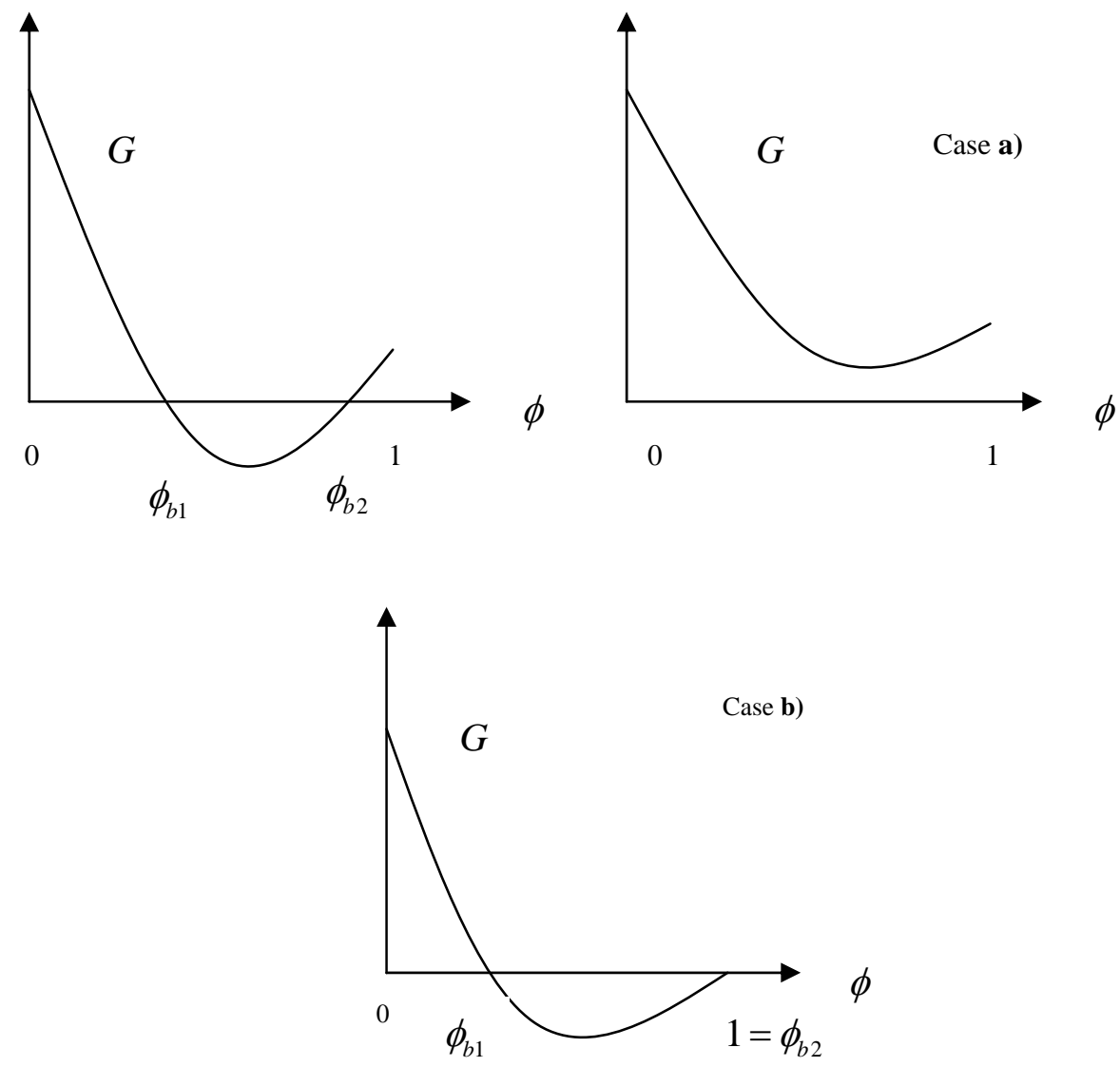

Figure 2. Analysis of the "break points"

Therefore, depending on how large the congestion parameter is, $G(\phi)=0$ has either two real roots - function $G$ cuts twice between 0 and $1-$ or it has not any $-G$ does not cut the axis at any point (case a). In the former case, it means that symmetry is a stable equilibrium for low and high values of $\phi$ (high and low transport costs, respectively), while in the latter an even distribution is always a stable equilibrium. It can be shown

\footnotetext{
${ }^{11}$ This is because of the aforementioned “no-black-hole condition”.
} 
that the minimum value of function $G$ increases with $\gamma$. Hence, the congestion parameter makes the curve rise, so that dispersion between both locations is more easily found. Numerical explorations of the above critical values suggest that the range of transport costs in which the symmetric equilibrium is stable expands with $\sigma$ and $H$, and shrinks with $\mu$ and $\mathrm{L}$. The effects of parameters $\sigma$ and $\mu$ are like those found in F-O, while the effects of the four parameters are in line with those obtained in the previous section.

In particular, if there were no congestion costs (case b), the two roots would be $\phi_{b 2}=1$ and

$$
\phi_{b 1}=\frac{(\mu-\sigma)(1+\mu-\sigma)}{(\mu+\sigma)(-1+\mu+\sigma)}=\frac{1-\mu / \sigma}{1+\mu / \sigma}\left(\frac{1-1 / \sigma-\mu / \sigma}{1-1 / \sigma+\mu / \sigma}\right),
$$

where the latter is identical to that obtained in F-O. This means that if transport costs are so large that $\phi$ is below the above threshold, symmetry between the two locations is a stable equilibrium; otherwise it is not. Therefore, we can obtain F-O's results as a particular case where $\gamma=0$.

It follows then that the inclusion of urban costs in the core-periphery model affects the range of transport costs within which the symmetric equilibrium is stable. In this vein, these costs make it possible not only to reach this spatial configuration at high but also at low transport costs (low and high values for $\phi$, respectively). 
Numerical examples

In order to illustrate the aforementioned results we depict the equilibria of the economy, as transport costs vary, for given values of the remaining parameters. ${ }^{12}$
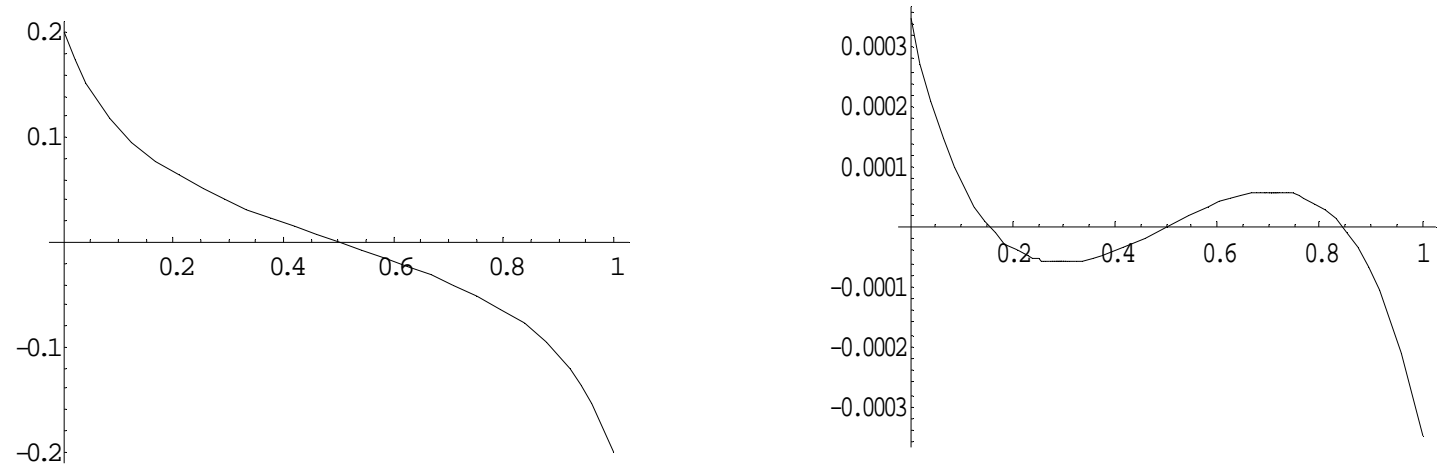

$\phi=0.1$

$\phi=0.665$
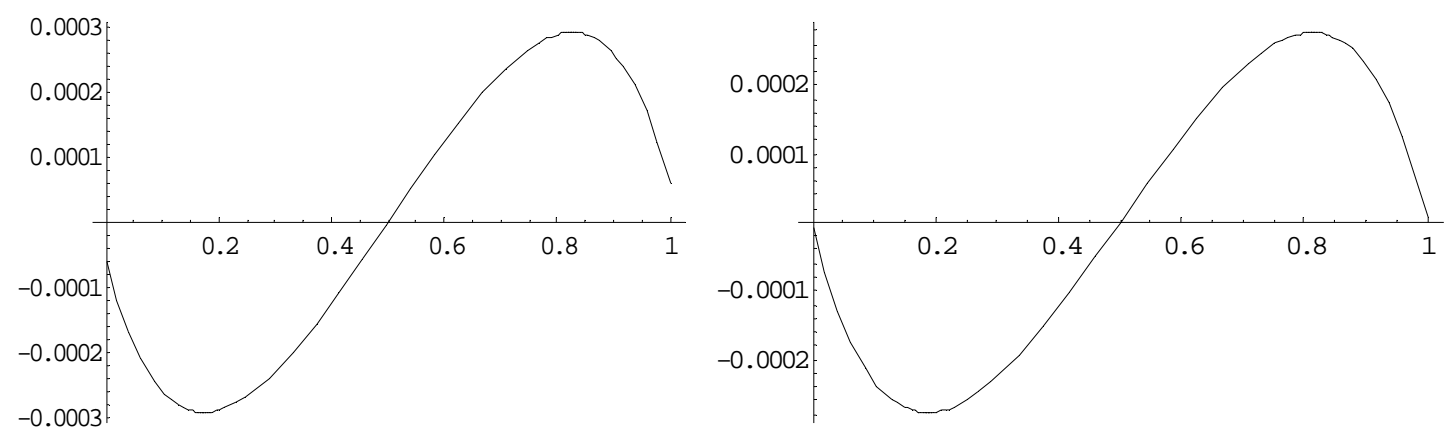

$\phi=0.7$

$$
\phi=0.8
$$
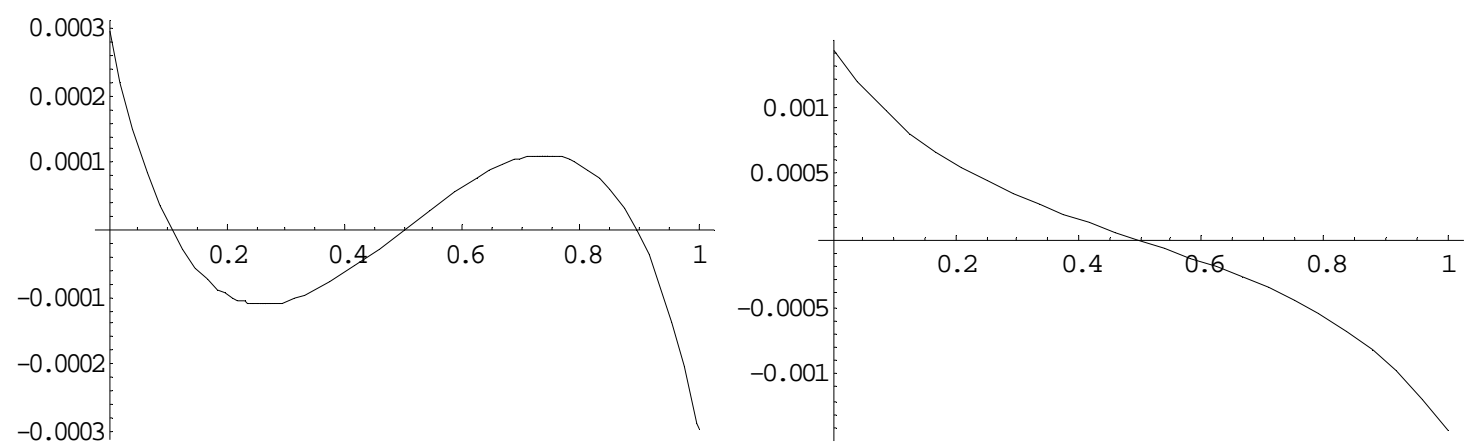

$\phi=0.83$

$$
\phi=0.9
$$

Figure 3. Real wage differential against $h$

12 In the figures these values are $\mu=0.5, \sigma=4, \gamma=0.05, \alpha=1, \beta=1, H=20, L=10$ and $L_{1}=L_{2}$. 
Figure 3 shows the real wage differential as a function of the proportion of high-skilled labor in City 1, for different transportation costs. It follows that both at high and low transport costs ( $\phi=0.1$ and 0.9 , respectively) the symmetric equilibrium is stable and unique, while at intermediate transport costs $(\phi=0.7$ and 0.8$)$ the respective concentration in each location are the only stable equilibria of the economy. Notice that the evolution from dispersion to concentration, and vice versa, is characterized by a gradual instead of a discontinuous change, so that asymmetric distributions of highskilled labor between both locations emerge as stable equilibria ( $\phi=0.665$ and 0.83 ). This gradual change is depicted in Figure 4, which shows the stable equilibria of the economy for the complete sequence of transport costs.

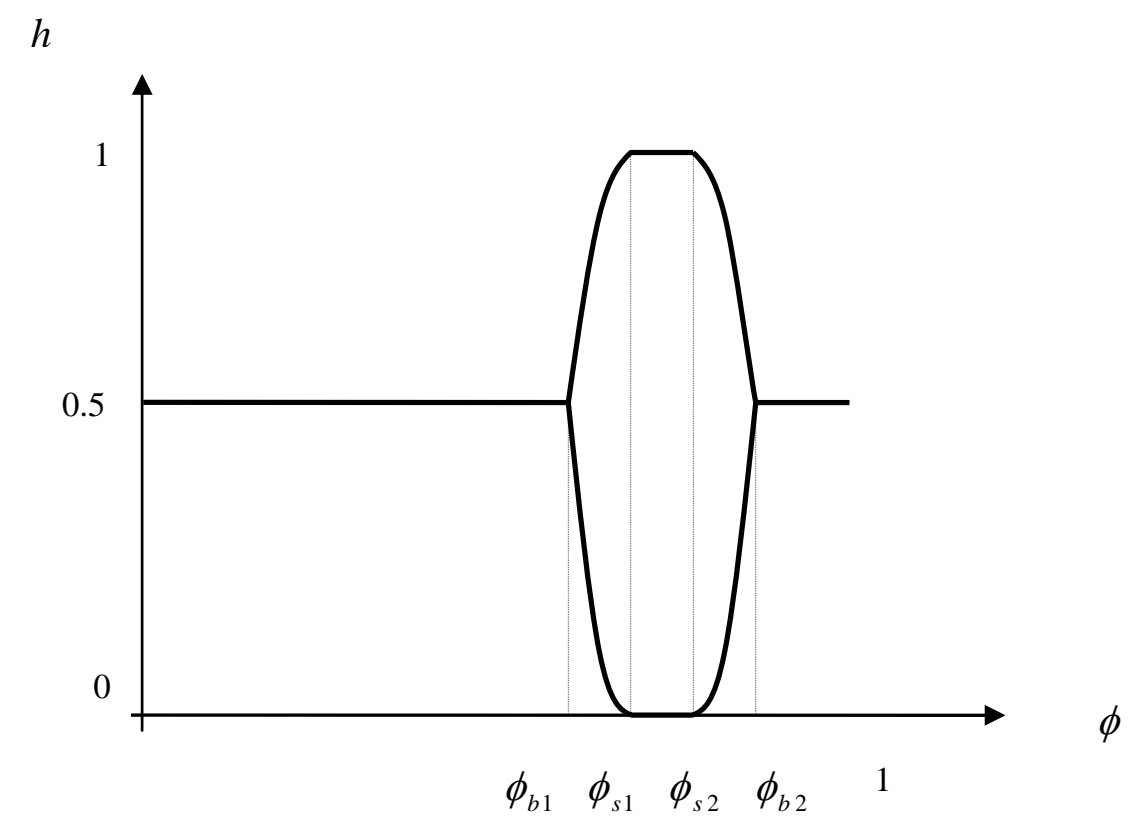

Figure 4. Stable equilibria

It follows that, unlike F-O, when considering not only the demand-pull effect but also congestion costs, we cannot warranty that full agglomeration and an even distribution of 
firms and workers are the only stable equilibria of the economy. ${ }^{13}$ On the other hand, and in line with the analytical results obtained in previous sections, concentration emerges just at intermediate transport costs. Figure 4 describes a relation between agglomeration and transport costs similar to that obtained by Puga (1999) in an alternative framework with vertical linkages between firms and labor immobility. In that paper, dispersion at low transport costs was caused instead by wage differentials between locations — which encouraged firms to move to the periphery- while here the existence of congestion costs is the centrifugal force driving to the same result.

When there are no congestion costs $(\gamma=0)$ the above figures become as follows:
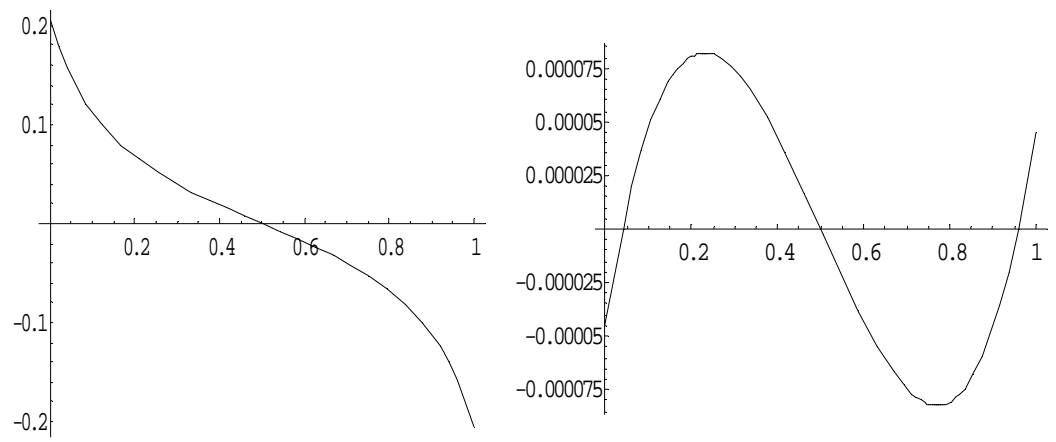

$\phi=0.1$

$\phi=0.551$
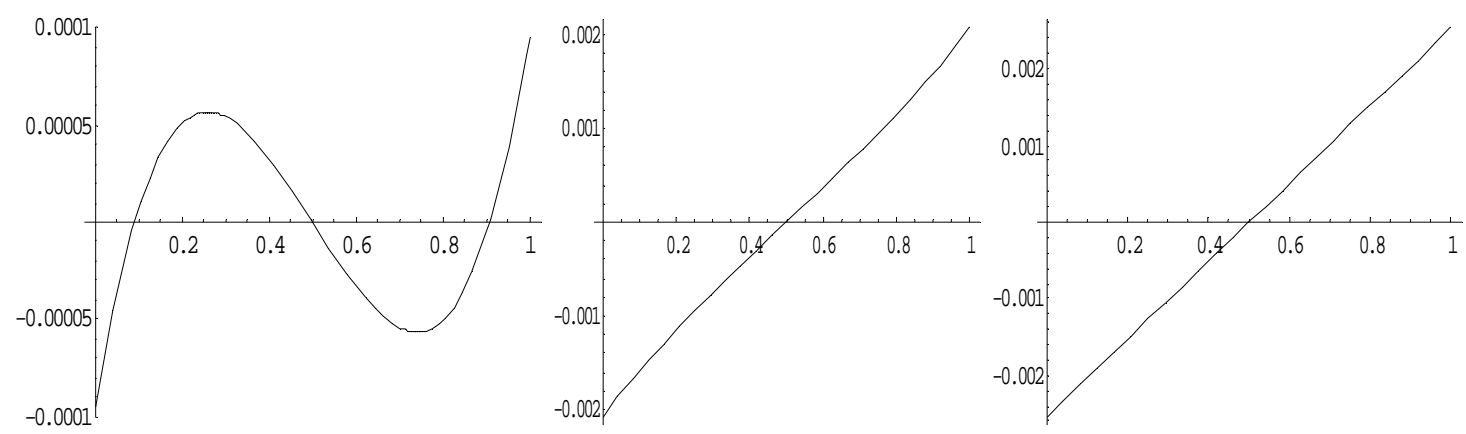

$\phi=0.552$

$\phi=0.6$

$\phi=0.9$

Figure 5. Real wage differential against $h, \gamma=0$

\footnotetext{
${ }^{13}$ It can be shown that $W(h, \phi)$ equals zero at most 5 times in the interval $h \in(0,1)$, four of these equilibria being symmetrically placed around $h=0.5$. See Appendix.
} 
There is an important difference with respect to Figure 3 when changing from low to high values of $\phi$, since asymmetric equilibria are now unstable. Then, the whole picture of stable equilibria for any given transport cost looks like:

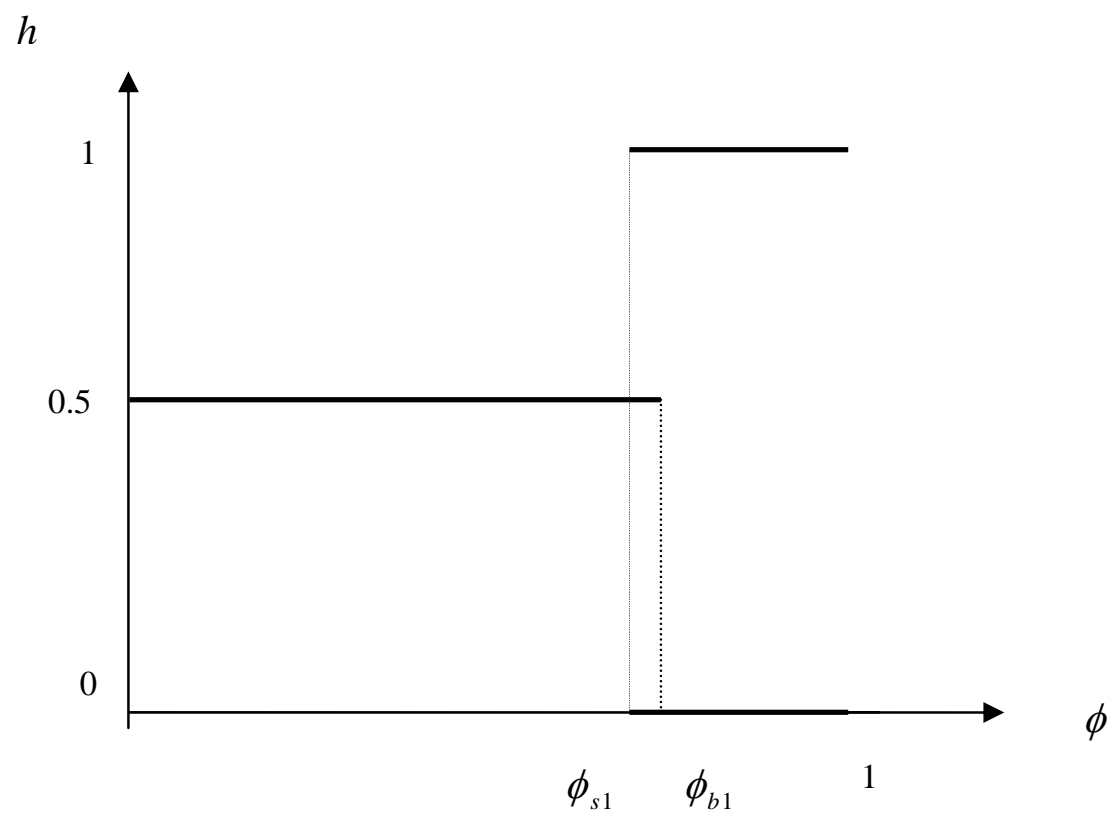

Figure 6. Stable equilibria, $\gamma=0$

By comparing Figures 4 and 6, three differences become evident. First, at high values of $\phi$, dispersion is possible if the are congestion costs. Second, while the change from dispersion to full agglomeration is gradual when considering congestion costs, otherwise it is not. Third, the relative position of sustain and break points reverses when there are no congestion costs. ${ }^{14}$

\section{Conclusions}

Firstly, we have analytically shown that, within the Dixit-Stiglizt-Iceberg framework, the symmetric equilibrium is stable not only at high but also at low transport costs,

\footnotetext{
${ }^{14} \mathrm{~F}-\mathrm{O}$ show that the sustain point is always lower than the break point.
} 
when considering the demand pull together with the effects of congestion costs. This result corroborates previous numerical simulations conducted by Tabuchi (1998). Secondly, the relationship between agglomeration and transport costs seems to be quite close to that found by Puga (1999) in an alternative model that considers labor immobility - together with input-output linkages between firms - and wage differentials between locations. It follows then that, at high transport costs, dispersion is caused by the demand pull, so that firms want to meet the immobile demand (either due to immobile rural markets or to international labor immobility). At low transport costs, dispersion is however caused by other centrifugal forces. In this regard, in Puga (1999), the periphery attracts firms because of its costs advantages, while in this model congestion cost is the effective centrifugal force at low transport costs. This suggests that the above relationship seems quite robust against alternative centrifugal forces jointly considered with the demand pull. ${ }^{15}$ Thirdly, unlike Puga (1999), we have shown that the mobile labor force increases the possibility of dispersion at low transport costs, while the immobile labor has the opposite effect. The differences between both papers can be explained as follows. In Puga (1999), labor mobility makes the centrifugal force that operates at low transport costs — that is, the wage differential — become ineffective. Here, instead, the mobile labor force is more closely related to congestion costs, so that the higher this mobile factor, the higher the role played by these urban costs. Fourthly, we have also shown that the inclusion of centrifugal forces other than the demand effect allows the emergence of asymmetric distributions of the economic activity and brings the possibility of a gradual change when switching from dispersion to agglomeration and vice versa.

\footnotetext{
${ }^{15}$ Note, however, that other assumptions regarding a given model can reduce the effect of a centrifugal force explicitly included in the analysis. So, for example, in Puga (1999), labor mobility can reduce the effect of the wage differential, so that dispersion cannot emerge as a stable equilibrium when transport costs are low if workers are geographically mobile.
} 


\section{References}

Forslid, R., Ottaviano, G. (2003) An analytically solvable core-periphery model. Journal of Economic Geography, 3: 229-240

Forslid, R., Haaland, J., Midelfart Knarvik, K. H. (2002) A U-shape Europe? A simulation study of industrial location. Journal of International Economics, 57: 273-297

Glaeser, E., Kohlhase, J. (2004) Cities, regions and the decline of transport costs. Papers on Regional Science, 83: 197-228

Harford, J. (2006) Congestion, pollution, and benefit-to-cost ratios of US public transit systems. Transportation Research Part D, 11: 45-58

Krugman, P. (1991 ) Increasing returns and economic geography. Journal of Political Economy, 99: 483-499

Krugman, P., Livas Elizondo, R. (1996) Trade policy and the Third World metropolis. Journal of Development Economics, 49: 137-150

Murata, Y, Thisse, J.-F. (2005) A simple model of economic geography à la HelpmanTabuchi. Journal of Urban Economics, 58: 137-155 
Ottaviano, G., Tabuchi, T., Thisse, J.-F. (2002) Agglomeration and trade revisited. International Economic Review, 43: 409-436

Picard, P., Zeng, D.Z. (2005) Agricultural sector and industrial agglomeration. Journal of Development Economics, 77: 75-106

Puga, D. (1998) The rise and fall of regional inequalities. European Economic Review, 43(2): 303-334

Robert-Nicoud, F. (2005) The structure of simple “New Economic Geography” models (or, On identical twins). Journal of Economic Geography, 5: 201-234

Tabuchi, T. (1998) Urban agglomeration and dispersion: A synthesis of Alonso and Krugman. Journal of Urban Economics, 44: 333-351

Tabuchi, T. Thisse, J.-F. (2002) Taste heterogeneity, labor mobility and economic geography. Journal of Development Economics, 69: 155-177

United Nations (2004) World Urbanization Prospects. The 2003 Revision. Data Tables and Highlights. Population division, Department of Economics and Social Affairs.

Venables, A. (1996) Equilibrium locations of vertically linked industries. International Economic Review, 37: 341-359 


\section{Appendix}

Set of interior equilibria

Indirect utility differential can be decomposed as:

$$
W(h, \phi)=A(h, \phi) \cdot V(h, \phi),
$$

where $^{16}$

$$
A(h, \phi)=\eta \frac{\mu L \alpha^{\frac{\mu}{1-\sigma}}(\sigma-1)^{\mu}}{2(\sigma-\mu) H^{\frac{1+\mu-\sigma}{1-\sigma}}(\beta \sigma)^{\mu}} \frac{1}{\phi\left[(1-h)^{2}+h^{2}\right]+\left[1-\frac{\mu}{\sigma}+\phi^{2}\left(1+\frac{\mu}{\sigma}\right)\right] h(1-h)}>0
$$

for any $h \in(0,1)$ and

$$
V(h, \phi)=\frac{2 \phi h+\left[1-\frac{\mu}{\sigma}+\phi^{2}\left(1+\frac{\mu}{\sigma}\right)\right](1-h)}{[h+\phi(1-h)]^{\frac{\mu}{1-\sigma}}\left(h H+\frac{L}{2}\right)^{\gamma \mu}}-\frac{2 \phi(1-h)+\left[1-\frac{\mu}{\sigma}+\phi^{2}\left(1+\frac{\mu}{\sigma}\right)\right] h}{[(1-h)+\phi h]^{\frac{\mu}{1-\sigma}}\left((1-h) H+\frac{L}{2}\right)^{\gamma \mu}} \equiv C(h)-C(1-h) .
$$

Note that $A(h, \phi)$ coincides with the expression found in F-O, while $V(h, \phi)$ is slightly different, since a new term —resulting from the congestion effect- appears. All interior equilibria satisfy that $V(h, \phi)=0$. If $C$ is a symmetric function, which may happen when there are no congestion costs $(\gamma=0)$ and $\phi=1, V(h, \phi)$ would be equal to zero for any $h$, so that there would be infinite equilibria. Otherwise, it can be shown that $\frac{\partial C}{\partial h}=0$ at most twice if $\gamma>0$, and at most once if $\gamma=0$, in the interval $(0,1)$. Given the symmetry between $C(h)$ and $C(1-h)$, it follows that function $V(h, \phi)$ is equal to zero at most five times if $\gamma>0$ and at most three times if $\gamma=0$. One of these equilibria is the symmetric one and the others are asymmetric equilibria placed symmetrically around it. This suggests that when including congestion costs in the model, there exists the possibility of stable equilibria other than the symmetric one, as shown in Figure 4.

${ }^{16} \eta \equiv \mu^{\mu}(1-\mu)^{1-\mu}$. 